\title{
Cardiovascular magnetic resonance can detect occult anthracycline cardiotoxicity in adolescents and young adults cancer survivors with normal ejection fraction
}

\author{
Miguel S Vieira ${ }^{1 *}$, Isma Rafiq ${ }^{1}$, Alberto C Figueroa ${ }^{2,1}$, Sujeev K Mathur ${ }^{4}$, Tarique Hussain ${ }^{3,1}$ \\ From 19th Annual SCMR Scientific Sessions \\ Los Angeles, CA, USA. 27-30 January 2016
}

\section{Background}

Risk stratification and early monitoring of anthracyclineinduced cardiotoxicity is crucial. It has been shown that early histopathological changes occur well before myocardial functional abnormalities can be detected. Additionally a long period of latency with clinical recovery of systolic function before the development of overt heart failure has been described, further emphasizing the importance of early recognition of cardiac injury and defining routine follow-up.

\section{Methods}

Long-term childhood cancer survivors were prospectively recruited after written informed consent. Study protocol was approved by the institutional review board. Subjects underwent a cardiovascular magnetic resonance (CMR) study on a 1.5 Tesla scanner (Philips, Best, Netherlands) with routine cine acquisition in long and short axis planes, tissue characterization with native $\mathrm{T} 1$ mapping and late gadolinium enhancement (LGE), selected 2D phase contrast flow imaging and 3D whole heart anatomy. Ventricular volumes, mass, and ejection fraction (EF) were obtained via standard planimetry techniques using a semiautomated commercial software (CMRtools, Imperial College, London, United Kingdom). Myocardial and left atrial (LA) strain parameters were derived from balanced SSFP cine images using dedicated software (TomTec Imaging Systems, 2D CPA MR, Cardiac Performance Analysis, Version 1.1.2.36, Unterschleissheim, Germany). LA reservoir, conduit and

'Division of Imaging Sciences \& Biomedical Engineering, King's College London British Heart Foundation Centre, London, United Kingdom Full list of author information is available at the end of the article contractile functions were then quantified by both fractional volume changes and CMR feature tracking derived strain and strain rate. Transit times for pulse wave velocity (PWV) computations were determined using the foot-to-foot methodology.

\section{Results}

Twenty patients (mean age $18 \pm 2$ years) were enrolled. Twenty age-matched healthy controls $(\mathrm{p}=0.109)$ were also recruited for comparison. Patients were studied after a mean anthracycline cumulative dose of $207 \mathrm{mg} / \mathrm{m}^{2}$, 83 months on average post-exposure. There was no significant difference between both groups in terms of volumetric analysis (table 1), with both presenting no areas of myocardial LGE. Left ventricular deformation parameters (but not LA functional indices) such as global radial strain (GRs) and strain rate (GRSr), global circumferential strain (GCs) and strain rate (GCSr), native T1 and PWV were significantly different among the two groups on both bivariate and multivariate regression analysis ( $\mathrm{p}<0.001$ to 0.035 ). GRSr (early diastole), GCSr (systole), PWV and native T1 outperform other imaging biomarkers (figure 1) in the assessment of subtle myocardial and vascular changes post-chemotherapy.

\section{Conclusions}

CMR can detect subtle myocardial and vascular abnormalities in young cancer survivors with normal EF exposed to anthracycline chemotherapy in the months following exposure. CMR may provide the short term markers of future cardiac morbidity necessary to design safer treatment regimens for younger cancer survivors. 
Table 1 Baseline characteristics

\begin{tabular}{|c|c|c|c|}
\hline CHARACTERISTICS & PATIENTS & CONTROLS & $p$ \\
\hline Age, years & $18 \pm 2[12-21]$ & $19 \pm 3[12-24]$ & 0.109 \\
\hline Gender, n & 11 males; 9 females & 12 males; 8 females & 0.749 \\
\hline LV ejection fraction [LVEF (\%)] & $59 \pm 3.5$ & $61 \pm 4.2$ & 0.057 \\
\hline LV end-diastolic volume indexed [LVEDVi $(\mathrm{mL} / \mathrm{m} 2)]$ & $85 \pm 16.5$ & $85 \pm 14.1$ & 0.975 \\
\hline LV end-systolic volume [LVESVi (mL/m2)] & $35 \pm 9.5$ & $35 \pm 9.1$ & 0.946 \\
\hline Global circumferential strain (GCs) & $-14.0 \pm 3.6$ & $-16.4 \pm 3.5$ & 0.041 \\
\hline Global radial strain (GRs) & $17.2 \pm 6.4$ & $30.7 \pm 6.9$ & $<0.001$ \\
\hline Global circumferential strain rate [GCSr (syst)] & $-1.25 \pm 0.26$ & $-1.58 \pm 0.41$ & 0.005 \\
\hline Global radial strain rate peak systole [GCRSr (syst)] & $0.86 \pm 0.21$ & $2.44 \pm 0.5$ & $<0.001$ \\
\hline Global circumferential strain rate early diastole [GCSr (diast)] & $1.49 \pm 0.41$ & $1.85 \pm 0.52$ & 0.022 \\
\hline Global radial strain rate early diastole [GRSr (diast)] & $-1.61 \pm 0.38$ & $-2.21 \pm 0.61$ & 0.001 \\
\hline Native $\mathrm{T} 1$ (mid septum, ms) & $1004 \pm 70.6$ & $966 \pm 30.6$ & 0.035 \\
\hline LA ejection fraction total (\%) & $66.9 \pm 8.3$ & $67.9 \pm 7.8$ & 0.692 \\
\hline LA conduit function (\%) & $41.2 \pm 15.1$ & $41.5 \pm 10.6$ & 0.928 \\
\hline LA booster pump function (\%) & $42.3 \pm 9.1$ & $44.5 \pm 10.8$ & 0.682 \\
\hline LA total strain $(\varepsilon s)$ & $30.6 \pm 8.6$ & $30.7 \pm 7.4$ & 0.967 \\
\hline LA passive strain ( $(\varepsilon e)$ & $24.1 \pm 6.4$ & $23.3 \pm 5.2$ & 0.638 \\
\hline LA active strain $(\varepsilon a)$ & $6.5 \pm 3.5$ & $7.5 \pm 3.6$ & 0.400 \\
\hline LA peak positive strain rate (SRs) & $1.2 \pm 0.3$ & $1.3 \pm 0.4$ & 0.430 \\
\hline LA peak early negative strain rate (SRe) & $-1.7 \pm 0.4$ & $1.7 \pm 0.4$ & 0.941 \\
\hline LA peak late negative strain rate (SRa) & $-0.9 \pm 0.3$ & $-1.0 \pm 0.3$ & 0.148 \\
\hline Pulse wave velocity [PW (m/s)] & $4.605 \pm 0.51$ & $3.742 \pm 0.23$ & 0.019 \\
\hline
\end{tabular}

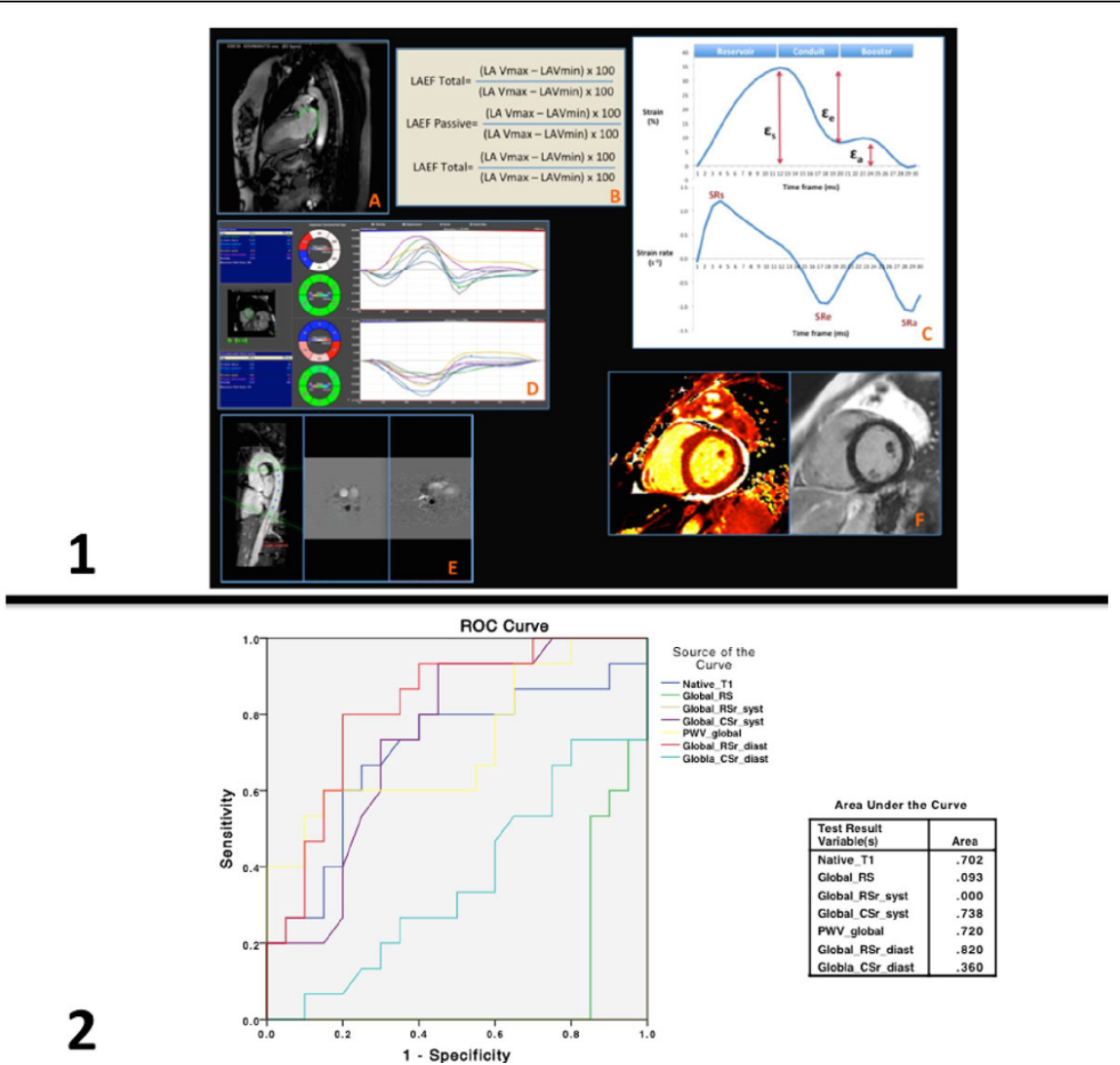

Figure 1 Panel 1 (A to F) depicts the PWV, T1 mapping/myocardial fibrosis analysis, LA and LV indices assessed. ROC curve (Panel 2) comparing the sensitivity and specificity of different imaging biomarkers analyzed and chemotherapy treatment. 


\section{Authors' details}

'Division of Imaging Sciences \& Biomedical Engineering, King's College London British Heart Foundation Centre, London, United Kingdom. ${ }^{2}$ Departments of Surgery and Biomedical Engineering, University of Michigan, Ann Arbor, MI, USA. ${ }^{3}$ Pediatric Cardiology, University of Texas, Dallas, TX, USA. ${ }^{4}$ Pediatric Cardiology, Evelina London Children's Hospital/ Guy's and St Thomas' NHS Foundation Trust, London, United Kingdom.

Published: 27 January 2016

doi:10.1186/1532-429X-18-S1-Q42

Cite this article as: Vieira et al:: Cardiovascular magnetic resonance can detect occult anthracycline cardiotoxicity in adolescents and young adults cancer survivors with normal ejection fraction. Journal of Cardiovascular Magnetic Resonance 2016 18(Suppl 1):Q42.

Submit your next manuscript to BioMed Central and take full advantage of:

- Convenient online submission

- Thorough peer review

- No space constraints or color figure charges

- Immediate publication on acceptance

- Inclusion in PubMed, CAS, Scopus and Google Scholar

- Research which is freely available for redistribution

Submit your manuscript at www.biomedcentral.com/submit 